\title{
The colonic bioreactor - a forerunner model for future biotechnology (function, role, products \& management)
}

\author{
Swidsinski A. \\ Humboldt University, Charite Hospital, Chariteplatz 1, 10098, Berlin, Germany, \\ e-mail: alexander.swidsinski@charite.de
}

doi: 10.36291/HIT.2019.swidsinski.017

Biotechnology is a key to our future. Using a bioreactor as a tool, men produce already nowadays gas, food, enzymes, insulin and a diverse set of biologicals on a very large scale. Adapting a biotechnology only on its own purpose, mankind is borrowing the wisdom of life, originating from four billion years of evolution. Actually, our earth itself is a large bioreactor, with a highly complex composition of different subsystems, each of them again specialized for a distinct purpose. Colon, is one of the most sophisticated natural bioreactors and as an organ indispensable for all vertebrates. Colonic bioreactor produces energy, vitamins, hormones, neuronal active substances and is important for antigen presentation purposes using a complex polymicrobial community. These functions are not a random result of accidentally crossing microbiota, but a result of deliberate selection, steering and facilitation of complex biomasses. We are just at the beginning of unravelling advanced morphologic and functional solutions.

The lecture describes the principles by which our colonic bioreactor is maintained, its relation to our health, functional disorders, inflammation, the interaction with antibiotics, probiotics and - last but not least - humic acids. Different to antibiotics, and probiotics, humic acids are extremely complex and still chemically poorly characterized. That is due to the complexity of the microbiota and substrates that humic acids are originating from. However, exactly this complexity stresses their highly differentiated role in microbe mediated organic synthesis and degradation, which can be potentially used for the specific regulation of polymicrobial metabolism within our bioreactor. 